\title{
Effects of Alcohol Abuse on Proliferating Cells, Stem/ Progenitor Cells, and Immature Neurons in the Adult Human Hippocampus
}

\author{
Tara Wardi Le Maître', Gopalakrishnan Dhanabalan', Nenad Bogdanovic ${ }^{2}$, Kanar Alkass' and Henrik Druid*,I \\ 'Forensic Medicine Laboratory, Department of Oncology-Pathology, Stockholm, Sweden; ${ }^{2}$ Neurogeriatric Clinic, Theme Aging, Karolinska University \\ Hospital, Stockholm Sweden
}

\begin{abstract}
In animal studies, impaired adult hippocampal neurogenesis is associated with behavioral pathologies including addiction to alcohol. We hypothesize that alcohol abuse may have a detrimental effect on the neurogenic pool of the dentate gyrus in the human hippocampus. In this study we investigate whether alcohol abuse affects the number of proliferating cells, stem/progenitor cells, and immature neurons in samples from postmortem human hippocampus. The specimens were isolated from deceased donors with an on-going alcohol abuse, and from controls with no alcohol overconsumption. Mid-hippocampal sections were immunostained for Ki67, a marker for cell proliferation, Sox2, a stem/progenitor cell marker, and DCX, a marker for immature neurons. Immunoreactivity was counted in alcoholic subjects and compared with controls. Counting was performed in the three layers of dentate gyrus: the subgranular zone, the granular cell layer, and the molecular layer. Our data showed reduced numbers of all three markers in the dentate gyrus in subjects with an on-going alcohol abuse. This reduction was most prominent in the subgranular zone, and uniformly distributed across the distances from the granular cell layer. Furthermore, alcohol abusers showed a more pronounced reduction of Sox2-IR cells than DCX-IR cells, suggesting that alcohol primarily causes a depletion of the stem/progenitor cell pool and that immature neurons are secondarily affected. These results are in agreement with observations of impaired adult hippocampal neurogenesis in animal studies and lend further support for the association between hippocampal dysfunction and alcohol abuse.

Neuropsychopharmacology (2018) 43, 690-699; doi:I0.1038/npp.2017.25I; published online I5 November 2017
\end{abstract}

\section{INTRODUCTION}

Previous research results indicate that the hippocampus is particularly susceptible to negative consequences of excessive alcohol consumption, thereby affecting learning and memory functions as well as emotional behaviors (Houser, 2007). An important feature of the adult hippocampus is its ability to continuously generate new neurons in the dentate gyrus (DG) area in rodents and monkeys (Altman and Das, 1965; Kornack and Rakic, 1999), a phenomenon implicated in maintaining hippocampal structure, integrity, and function (Kempermann, 2002). Eriksson et al (1998) were the first to report that new neurons are generated in the adult human hippocampus. In the hippocampus of adult humans and nonhuman primates, 5-bromo-2-deoxyuridine (BrdU) immunoreactivity is found in the molecular layer (ML), the inner part of the granular cell layer (GCL), the subgranular zone (SGZ), and the CA4 (Eriksson et al, 1998; Kornack and Rakic, 1999).

* Correspondence: Professor H Druid, Forensic Medicine Laboratory, Department of Oncology-Pathology, Retzius väg 3, SE-17I 77, Stockholm, Sweden, Tel: +46 (0)8 425877 70,

E-mail: henrik.druid@ki.se

Received 21 March 2017; revised 12 October 2017; accepted 16 October 2017; accepted article preview online 20 October 2017
Adult neurogenesis has been studied in experimental animals by utilizing numerous biomarkers representing different stages of the neurogenesis process. Among these markers, Ki67, SRY-related HMG box 2 (Sox-2), and doublecortin (DCX), have frequently been used in studies of neurogenesis. Ki67 is an endogenous marker of proliferation that is expressed throughout the active phases of cell division except G0 (Scholzen and Gerdes, 2000). Sox-2 is expressed by stem/progenitor cells and astroglia in the adult rat brain (Komitova and Eriksson, 2004). Sox2 controls proliferation and maintenance of the undifferentiated neural stem cell (NSC) state (Schwarz et al, 2012). DCX is a brainspecific microtubule-associated protein that is necessary during migration of immature neurons and might have a role in neurite development (Friocourt et al, 2003; Gleeson et al, 1999). The anti-NeuN antibody has been widely used as a marker of postmitotic neurons (Mullen et al, 1992). Recently, a unique study by Spalding et al (2013) using a novel retrospective birth dating strategy that utilizes bombpulse ${ }^{14} \mathrm{C}$ incorporation into the DNA of NeuN-positive cells showed that hippocampal neurons are generated at comparable rates in the middle-aged humans and mice, strongly suggesting a functional importance of neurogenesis in adult humans. 
Alterations in adult neurogenesis in the hippocampal formation has been linked to diseases such as Alzheimer's, depression, schizophrenia, and to the development of addiction, including alcoholism (Bayer et al, 2015; Boldrini et al, 2013; Nixon and Crews, 2002; Reif et al, 2006).

Hippocampus is critical for aspects of learning and as such is implicated in the acquisition, consolidation, and expression of context-dependent drug memories (Hyman et al, 2006). Many researchers therefore now postulate a role of hippocampus in drug seeking and relapse (Belujon and Grace, 2011; Vorel et al, 2001). Furthermore, animal experiments have shown that alcohol exposure decreases adult neurogenesis in the hippocampal formation (He et al, 2005; Nixon and Crews, 2002; Taffe et al, 2010) affecting most of the stages of neurogenesis: cell proliferation, neuronal differentiation, cell migration, survival, and circuitry integration (Nixon, 2006). We have been unable to find any studies specifically focusing on alcohol and hippocampal neurogenesis in humans. However, in a study on deceased patients with schizophrenia, depression, and controls, Reif et al (2006) mentioned that they did not find lower numbers of Ki67-positive cells in hippocampus in subjects with alcohol abuse, but they did not provide details of the subjects. We therefore sought to test the hypothesis that alcohol abuse decreases the numbers of proliferating cells, stem/progenitor cells, and/or immature neurons in the human dentate gyrus. To this end, we decided to investigate the effects of alcohol abuse on the expression of Ki67, Sox2, and DCX in hippocampi from deceased alcoholics and control subjects. The selection of biomarkers was based on previous rodent, nonhuman primate, and human studies of neurogenesis (Boldrini et al, 2009; Crews et al, 2010; Knoth et al, 2010; Komitova and Eriksson, 2004; Mathews et al, 2010; Mullen et al, 1992; Reif et al, 2006; Taffe et al, 2010; Winner et al, 2012).

\section{MATERIALS AND METHODS}

\section{Case Selection}

The brain samples were collected from deceased persons subjected to a forensic autopsy at the Department of Forensic Medicine in Stockholm by KI Donatum, a core facility at Karolinska Institutet providing postmortem samples from well-characterized deceased donors for research purposes. Information about the subjects was obtained by perusing autopsy protocols, police reports, medical records, and from interviews with the relatives.

Identification of severe alcohol abusers and controls is fairly straightforward using information from the police, relatives, medical records, and postmortem findings, including microscopy and toxicology. Further characterization of subjects regarding drug use and somatic and psychiatric illnesses was based on semistructured interviews with relatives. Cases with severe depression, epilepsy, or brain injuries were excluded, as well as cases with pharmaceutical or illicit drug abuse. Supplementary Table S1 provides information on age, gender, cause of death, postmortem interval (PMI), brain $\mathrm{pH}$, alcohol and drug use, and presence of depression and liver pathology. In total, 35 cases, 28 males and 7 females, aged $24-78$ years were included in the study; 18 alcoholic and 17 control subjects. Mean age was 55 (median 54) and 54 (median 57) years for alcoholic and control subjects, respectively. Median PMI was $41 \mathrm{~h}$ (range $5.25-64 \mathrm{~h}$ ) and $40.5 \mathrm{~h}$ (range $11.25-84.5 \mathrm{~h}$ ) for alcoholics and controls, respectively. Median warm time (time before cold storage at $4^{\circ} \mathrm{C}$ ) was $6 \mathrm{~h}$ for alcoholics (range $2-19 \mathrm{~h}$ ) and $7 \mathrm{~h}$ for controls (range $2-41 \mathrm{~h}$ ).

\section{Inclusion Criteria for the Alcoholic Group}

For this study it was critical that the deceased donors had an on-going alcohol abuse as neurogenesis might be restored during (prolonged) periods of abstinence as observed in animal studies (He et al, 2009). Furthermore, studies of human alcoholics have shown that alcoholic sobriety results in improved brain function and regained volume (Sullivan et al, 2000). These functional changes may partly be explained by a restoration of neurogenesis (Crews et al, 2005). We therefore decided to only include alcoholic subjects, who either had been consuming large amounts of alcohol for at least 4 weeks before death or were known severe alcoholics with at least repeated binge drinking episodes with severe drunkenness during this time. The choice of the 4-week time window is also based on studies in nonhuman primates showing that at 6 weeks $84 \%$ of all the BrdU-labeled cells were DCX positive (Kohler et al, 2011). We therefore considered 4 weeks of alcohol abuse a suitable time period for the evaluation of alcohol's effects on the densities of the markers of proliferating cells, stem/ progenitor cells, and immature neurons, and still short enough to be able to obtain reasonably accurate information about drinking history.

\section{Sample Preparation and Immunofluorescence}

Left hippocampus was dissected free from adjacent structures, and a $4-5 \mathrm{~mm}$ thick coronal block was collected from the mid-portion, hence at the border between anterior and posterior hippocampus, at the level of corpus geniculatum laterale (Figure 1a). The block was immersed in a mixture of isopentane and dry ice at $-35^{\circ} \mathrm{C}$ to minimize structural artifacts, and then stored at $-80^{\circ} \mathrm{C}$ until cryosectioned and processed for immunohistochemistry. Coronal sections of $20 \mu \mathrm{m}$ thickness were prepared and mounted on electrostatically modified Superfrost Plus (Thermo Scientific) slides using a MICROM HM 500M (MICROM International $\mathrm{GmbH})$ cryostat at $-20^{\circ} \mathrm{C}$. The sections were air dried at room temperature (RT), rehydrated in phosphate buffer saline (PBS) ( $\mathrm{pH} \mathrm{7.7),} \mathrm{and} \mathrm{fixed} \mathrm{in} 4 \%$ formaldehyde in PBS for $15-30 \mathrm{~min}$. Sections were subsequently incubated for $1 \mathrm{~h}$ in blocking solution containing $1 \%$ BSA, 0.3\% Triton-X, $0.01 \% \mathrm{NaN}_{3}$, and normal donkey serum (NDS) in PBS at RT. After blocking, sections were incubated with primary antibodies (mouse anti-Ki67 (1:250, Dako), mouse antiNeuN (1:1000, Millipore), guinea pig anti-DCX (1:1000, Millipore), and rabbit anti-Sox2 (1:250, Millipore)) overnight at $4{ }^{\circ} \mathrm{C}$. Sections were then incubated with the following conjugated secondary antibodies: anti-mouse Alexa Fluor 488 (1:500, Invitrogen), anti-rabbit Alexa Fluor 555 (1:500, Invitrogen), or anti-guinea pig Cy3 (1:300, Jackson ImmunoResearch). The nuclei were visualized with Hoechst 33342 (0.2 $\mu \mathrm{l} / \mathrm{ml}$, Molecular Probes). 

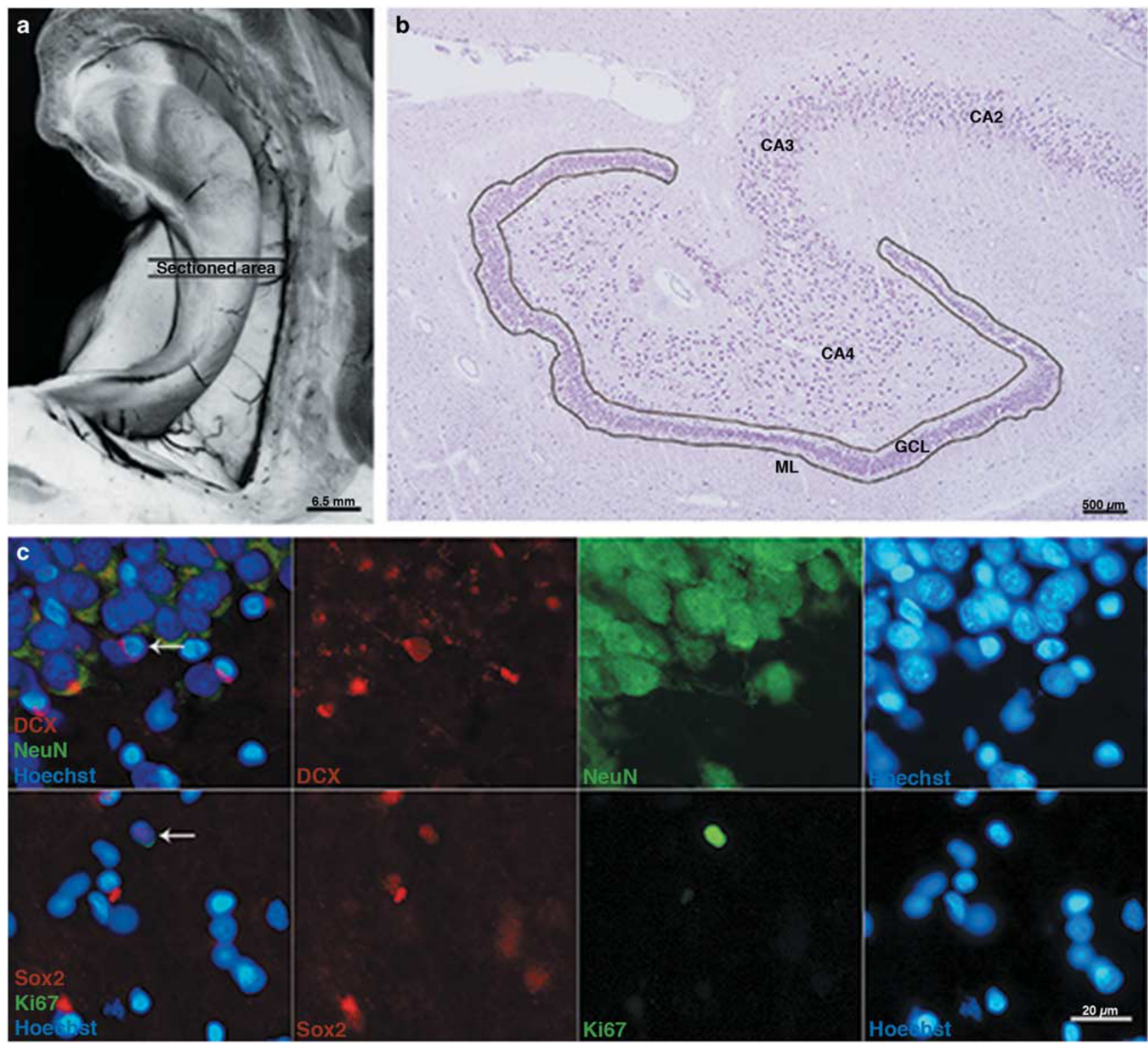

Figure I (a) Rostral view of the human hippocampus showing the area examined. (b) Low-power micrograph of one section of the human DG (antiNeuN staining). The outline defines the region of interest within which cells positive for Ki67, Sox2, and DCX were counted. (c) Representative photomicrographs of markers used in this study. In the upper panel, coronal sections of the human hippocampus were immunostained for DCX (red) and NeuN (green), and cell nuclei were labeled with Hoechst (blue, upper and lower panels). A high percentage of coimmunoreactivity between DCX and NeuN was observed in this study. The lower panel shows Sox2-IR (red) and Ki67-IR (green). Coexpression between Ki67 and Sox2 was sporadic. The arrows in the merged micrographs to the left in both panels show a cell coexpressing the markers of interest.

\section{Quantification of Markers of Proliferation, Stem/ Progenitor Cells, and Immature Neurons}

Sections were examined for Ki67 immunoreactivity (Ki67IR) (17 controls and 18 alcoholics); Sox2 (Sox2-IR) immunoreactivity (16 controls and 16 alcoholics); and DCX immunoreactivity (DCX-IR) (15 controls and 16 alcoholics) in all layers of DG. Approximately every tenth section was stained and an average of 5 sections for each case and staining were used. Stained sections were analyzed using a Nikon Eclipse E400 epi-fluorescence microscope (Nikon, Japan) equipped with a camera unit (Nikon Digital sight: DS-2Mv, DS-U2), UV, rhodamine, FITC, and merge filter setup, and using the NIS-elements BR 2.30 software (Nikon,
Japan). Positively stained cells were identified on the basis of cellular and nuclear morphology, and staining pattern. Cells with lipofuscin accumulation, the aging-related macular degeneration deposits of lipids and proteins (Terman and Brunk, 2004) were excluded.

The number of DCX-IR, Sox2-IR, and Ki67-IR (Figure 1c) cells were determined along the entire length of the GCL. Positive cells were counted throughout the DG, including the $\mathrm{ML}$, the GCL, and the SGZ (Figure $1 \mathrm{~b}$ ) and results were expressed as the number of immunoreactive cells per total GCL area $\left(\mathrm{mm}^{2}\right)$ of all sections examined in each case to overcome area differences between cases and sections. This value is referred to as the marker (Ki67, Sox2, DCX) index. As only the mid-portion of the border between anterior and 
posterior portion of the left hippocampus was used in this study, we have applied a two-dimensional quantitative approach (Bayer et al, 2015) with respect to unit area of GCL as reference rather than using volume as in stereological studies. The use of the GCL area as a reference was chosen to minimize error due to oblique cutting that is inevitable because of the irregular structure of the DG. Even though a 3D approach would have been desirable, the low number of positive cells makes an appropriate stereology study very difficult. Hence, whenever densities or numbers appear in this article, both terms refer to the index values according to the definition above.

In the absence of a clear delimitation of the SGZ in the adult human hippocampus, we arbitrarily used a distance of $100 \mu \mathrm{m}$ from the border of the GCL as the outer limit of this zone. We also applied the same limit for the ML, and the DG was consequently defined as the GCL and both these layers with $100 \mu \mathrm{m}$ width (Figure 1b) (for details see Discussion). Manual counting was performed using an eyepiece graticule at $\times 40$ magnification and the observer was blinded to the group. For each cell, the distance to the border of the GCL was measured using a dynamic scale device provided by the NIS Element software. Weak signals were studied with a $\times 60$ dry objective when necessary. Counting was performed under blinded conditions by two observers.

\section{Data Analysis}

GraphPad prism software 6.05 (GraphPad Software, USA) and IBM SPSS Statistics version 23 (IBM USA) were used for statistical analysis. We used the index value of each case and compared alcohol with control groups to investigate the effect of alcohol on adult neurogenesis. Comparisons between groups regarding all markers examined in these experiments were analyzed using the Mann-Whitney $U$-test. Spearman's rank correlation was used to analyze relationship between each marker's index values and continuous variables. A $p$-value of $<0.05$ was considered significant.

\section{Ethical Considerations}

The study was approved by the Regional Ethical Review Board in Stockholm, Dnr 2010/313-31/3. For all cases, consent was either obtained from the Swedish Donation registry, donation card, or from next of kin. In either case, the family was always informed about the project and was given reasonable time to consider the decision. This procedure was handled by KI Donatum. The relatives were also offered to participate in a semistructured interview that typically took place within a few days after the first contact. After completion of the data extraction, the cases and their related data were pseudonymized.

\section{RESULTS}

\section{Ki67-IR in Alcohol Abusers and Control Subjects}

Ki67-IR was found in all layers (ML, GCL, and SGZ) of DG. The positivity was always strong and conspicuous. In both alcoholics and controls the expression of Ki67-IR was sparse.

We found that Ki67-IR was significantly reduced in the DG of alcoholics $(p=0.0011$; Figure $2 \mathrm{a})$ and in the SGZ
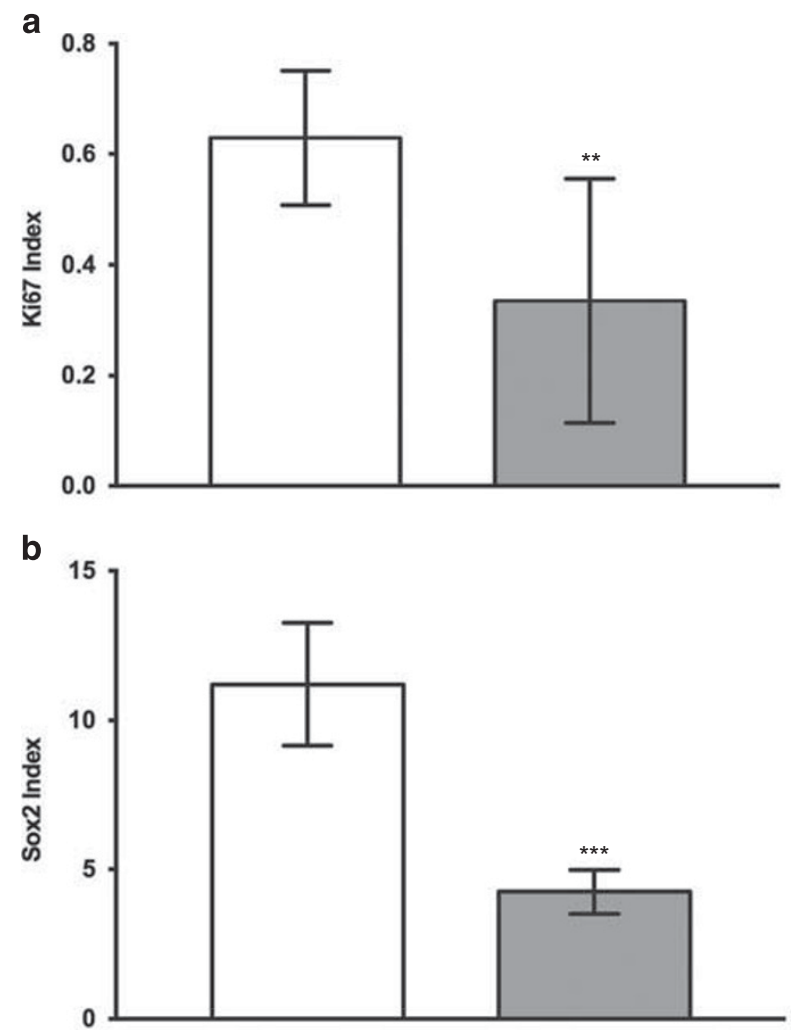

C

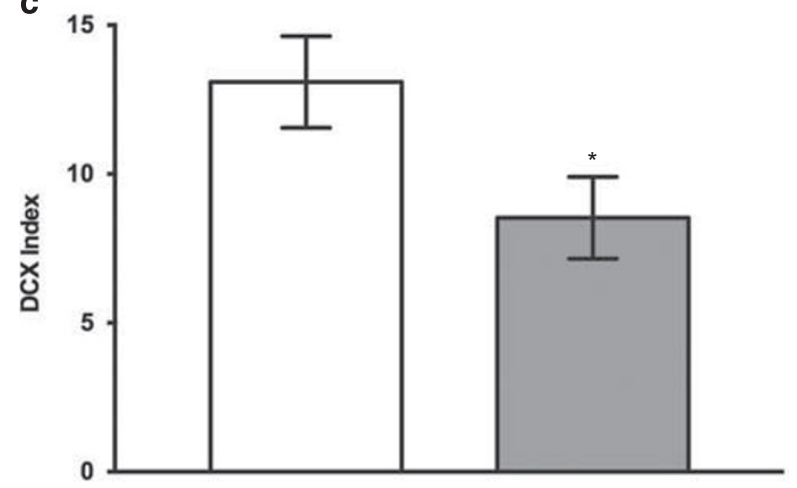

Figure 2 Immunopositivity of markers in control and alcoholics per unit area of GCL $\left(\mathrm{mm}^{2}\right)$ in DG: index values for (a) Ki67 ( $n$ (controls) $=17$ and $n$ (alcoholics) = 18), (b) Sox2 ( $($ controls) $=16$ and $n$ (alcoholics) $=16)$, and (c) DCX (n (controls) $=15$ and $n$ (alcoholics) =16) were significantly reduced in alcoholics as compared with controls. The index value is the number of positively stained cells per $\mathrm{mm}^{2}$ of GCL. Data are expressed as mean index value \pm SEM; $* p<0.05$, *** $p<0.01$, **** $p<0.001$ (MannWhitney U-test).

specifically ( $p=0.0068$; Figure $3 \mathrm{a})$ (Mann-Whitney $U$-test) compared with controls.

\section{Sox2-IR in Alcohol Abusers and Control Subjects}

Sox 2 was found in all layers of DG. The immunoreactivity was almost exclusively present as scattered, distinct spots within the cell nuclei (see Supplementary Figure S5), similar to the pattern previously reported for the same antibody (Knoth et al, 2010). Sox2 index values were significantly lower in alcoholics as compared with controls in the DG $(p=0.0005 ;$ Figure 2b, Mann-Whitney $U$-test $)$, and 


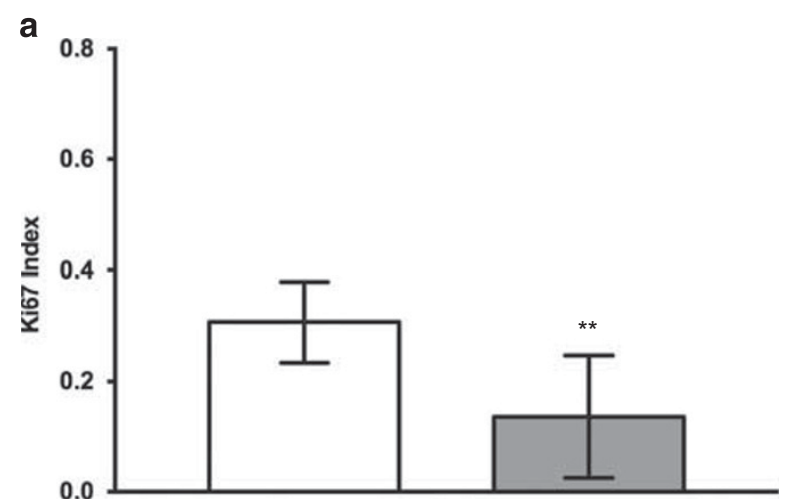

b

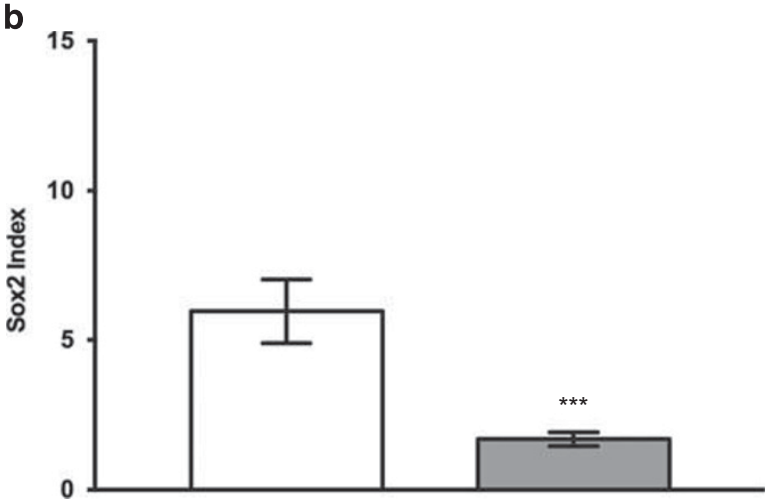

C

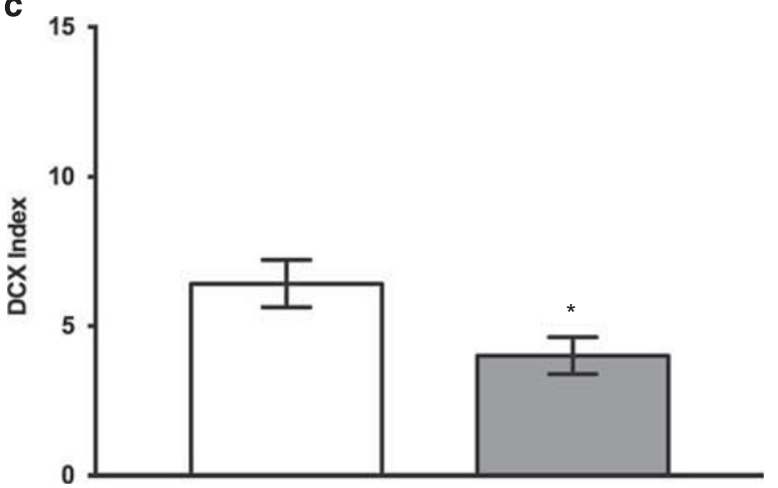

Figure 3 Immunopositivity of markers in control and alcoholics per unit area of GCL $\left(\mathrm{mm}^{2}\right)$ in SGZ: index values for Ki67 (a), Sox2 (b), and DCX (c) were significantly reduced in alcoholics as compared with controls. The index value is the number of positively stained cell counts per $\mathrm{mm}^{2}$ of GCL. Data are expressed as mean index value \pm SEM; ${ }^{*} p<0.05$, ** $p<0.0$ I, **** $p<0.00$ I (Mann-Whitney U-test).

specifically in the SGZ ( $p=0.0003$; Figure $3 \mathrm{~b}$ ). In some of the cases, a double staining for Sox 2 and Ki67 was applied. Only a very small proportion of the positive cells showed positivity for both markers. Almost all Sox2-IR cells within the three areas examined were negative for the glial fibrillary acidic protein (GFAP) (Supplementary Figure S5). However, the coexpression of these two markers was only studied in a limited number of sections.

\section{DCX-IR in Alcohol Abusers and Control Subjects}

DCX-IR cells were also found in all areas examined. The immunoreactivity was typically found in the cell soma and only occasionally in the processes (see Supplementary
Figure S5), and most of the cells were also positive for NeuN; and hence the same staining pattern as we previously have observed with the same antibody in striatum and hippocampus (Ernst et al, 2014). We found that the DCX index was significantly reduced in alcoholics compared with controls in the DG $(p=0.0240$, Mann-Whitney $U$-test; Figure 2c) and specifically in the SGZ ( $p=0.0215$; Figure $3 c)$.

In the GCL, there were no differences between groups for any of the markers, whereas a reduction of Sox2-IR and DCX-IR cells was observed in the ML (Supplementary Figures S1 and S2).

\section{Topographical Distribution of Ki67-, Sox2-, and DCX-IR}

The analysis of the frequency of positive immunoreactivity of all the markers at different distances from the GCL in the ML and the SGZ in control subjects revealed that the counts did not gradually decrease with increasing distance from the GCL as we originally expected. Instead, the immunoreactivity was fairly uniform at different distances with interspersed lows and highs (Figure 4). However, when exploring areas $>100 \mu \mathrm{m}$ from the ML and GCL, the immunoreactivity gradually became sparser, particularly for Sox 2 and DCX (no systematic counting was performed). We compared the distribution of the markers within $100 \mu \mathrm{m}$ distance and found that Ki67-IR and Sox2-IR cells were localized at longer distances from the GCL than DCX-IR cells; 68 and $55 \mu \mathrm{m} v s$ $49 \mu \mathrm{m}$, respectively, in control and alcoholic subjects (Supplementary Table S2). The immunoreactivities for all three markers were significantly reduced in alcoholics as compared with controls (Figure 2). This reduction was fairly evenly distributed across all distances from the GCL (Figure 4 and Supplementary Figure S3). The distance from the inner border of GCL to the border of CA4 (see Supplementary Figure S5A) was not different in cases with high and low index values (data not shown), implying that the reduction in cell densities in alcoholics cannot be explained by increase in neuropil volume.

\section{Possible Confounding Factors}

As DCX-positive cells in the SGZ previously has been reported to decrease slightly with age (Knoth et al, 2010), we analyzed the possible correlation between each marker's index values and age (both groups taken together, and separately). Ki67-IR significantly decreased with age in the total cohort and in the control group $(p=0.0242$ and $p=0.0470$, respectively, Spearman's rank correlation), but did not reach significance in the alcoholics. None of the other comparisons showed any statistically significant reduction with age. The scatter plots of index values $v s$ age of subjects in the SGZ presented in Figure 5 rather highlight an interindividual variation, but also show that on-going alcohol abuse seemingly reduces hippocampal neurogenesis, as estimated from the markers used, regardless of the individual's age. Ki67-IR was also significantly reduced with age in the DG of controls $(p=0.0235$; Supplementary Figure S4).

Although cases with severe depression were excluded, we did include four subjects (one control and three alcoholics) with reportedly mild depression during the 4 weeks before death, two of whom were treated with antidepressants, and two without medical treatment. Exclusion of these subjects 


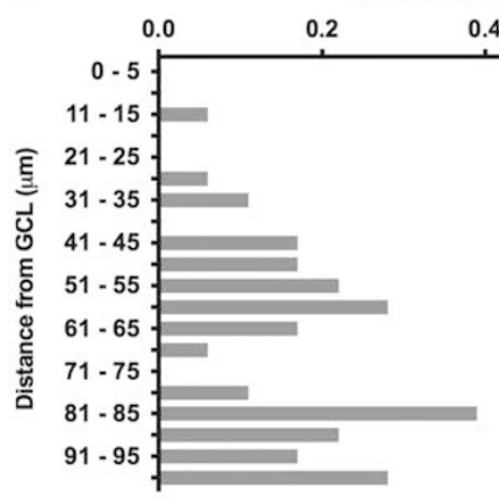

C

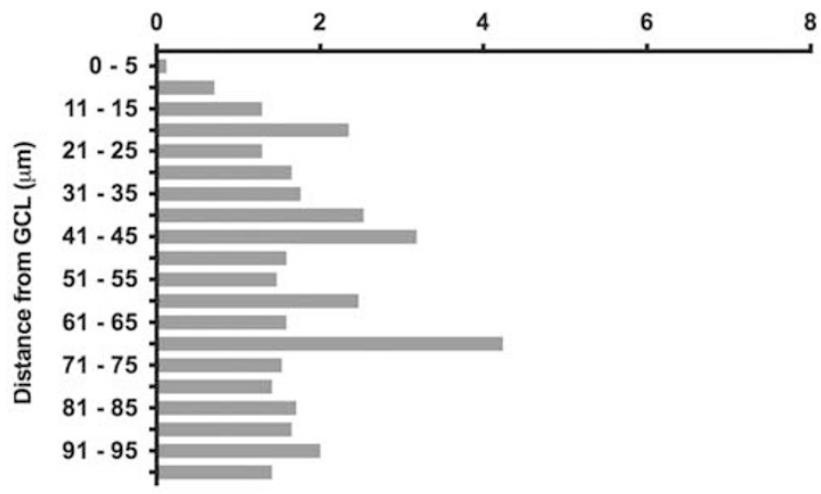

e

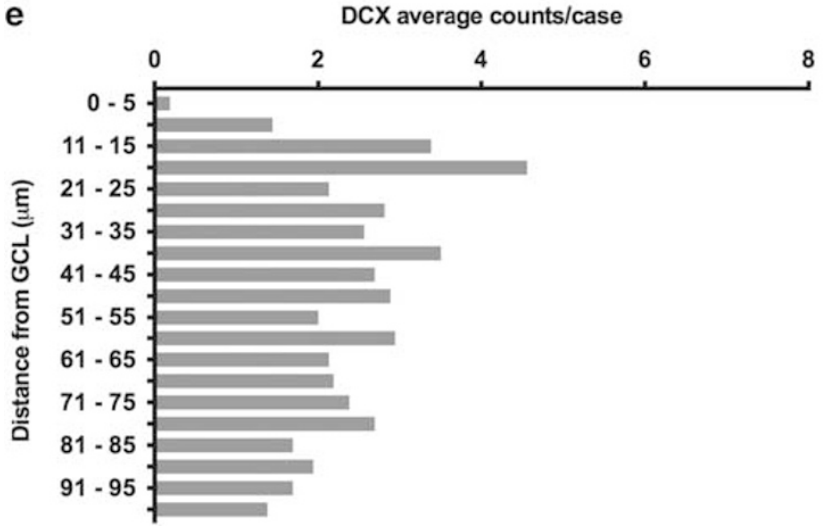

b

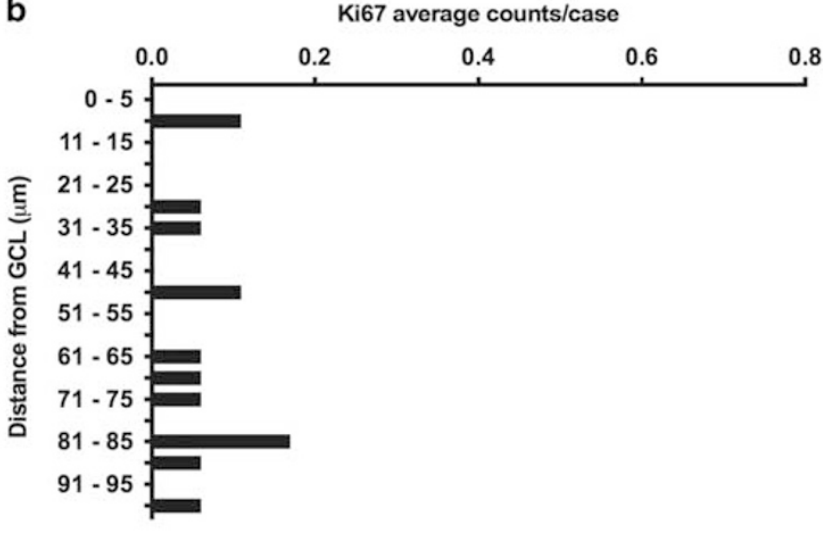

d

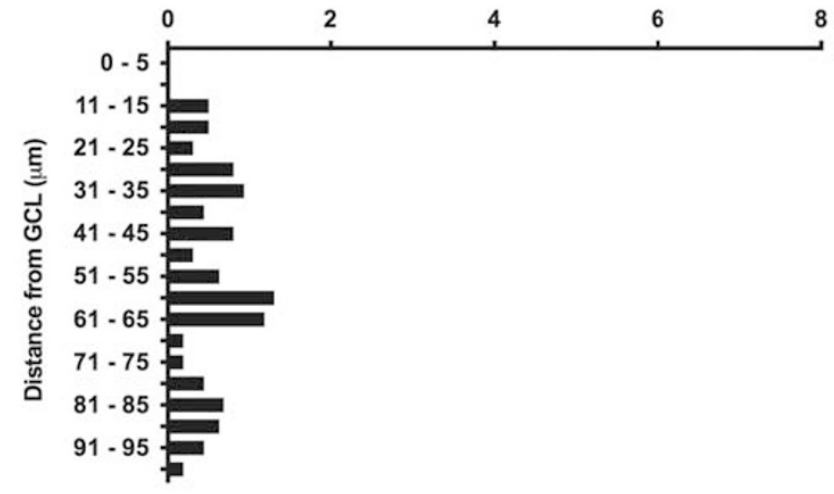

f

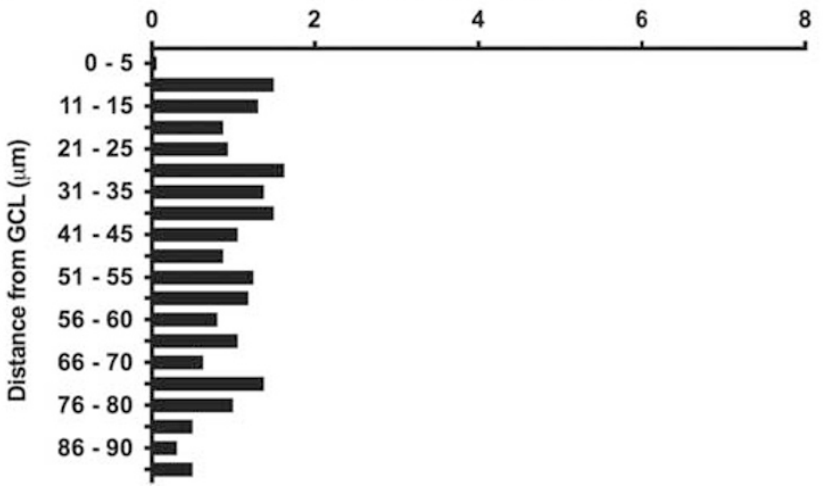

Figure 4 Distribution of immunoreactivity of markers used in the SGZ at different distances from the GCL: distribution of Ki67-IR cells/case in controls (a) and alcoholics (b), Sox2-IR cells/case in controls (c) and alcoholics (d), respectively; DCX-IR cells/case in controls (e) and alcoholics (f), respectively.

did slightly change the $p$-values, but not the statistical significance levels.

There were no significant differences between alcoholics and controls regarding brain weight, warm time, or total PMI. However, brain $\mathrm{pH}$ (measured in cerebellum samples) was lower in alcoholics as compared with controls. The difference in $\mathrm{pH}$ might be explained by some degree of alcoholic ketoacidosis among the alcoholics, although no analysis for $\beta$-hydroxybutyrate or acetoacetate was performed. However, there was no correlation between marker index in DG and $\mathrm{pH}$ for any of the markers, and therefore we do not think that the tissue $\mathrm{pH}$ technically has affected the densities of markers used in this study. Furthermore, there was no correlation between any of the marker index values and warm time, total PMI, or brain weight.

\section{DISCUSSION}

In this study, we show that on-going alcohol abuse is associated with a reduced number of Ki67-IR, Sox2-IR, and DCX-IR cells in the human DG. Our data represent a quantitative estimate of proliferating cells, stem/progenitor cells, and immature neurons in the DG from wellcharacterized human alcoholics with an on-going alcohol abuse and from control subjects. Ki67-IR, Sox2-IR, and DCX-IR were counted in the DG consisting of the GCL and $100 \mu \mathrm{m}$ of each of the SGZ and the ML. This width of the areas of interest constitutes a compromise between very thin and very wide zones previously examined. Whereas Eriksson et al (1998) have studied the occurrence of BrdU neurons in the SGZ within two neurons width from the GCL, Boldrini 


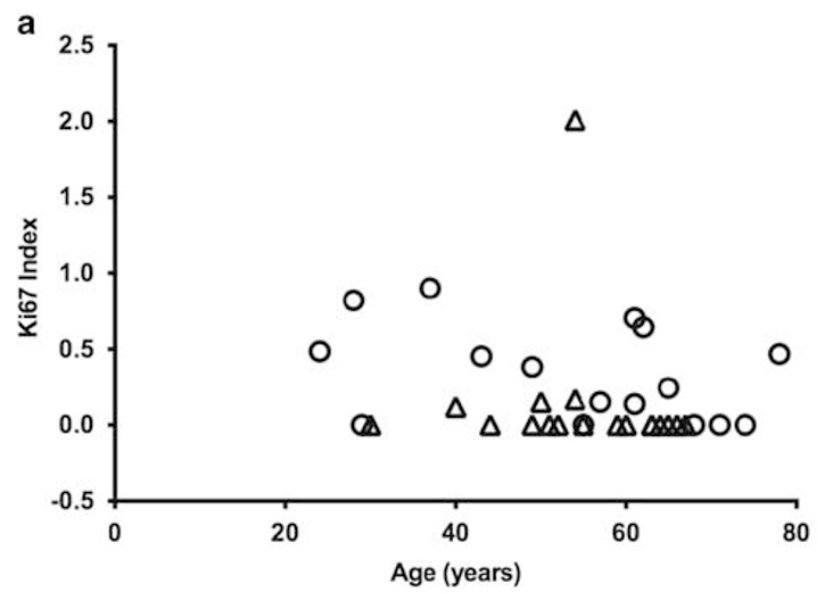

b

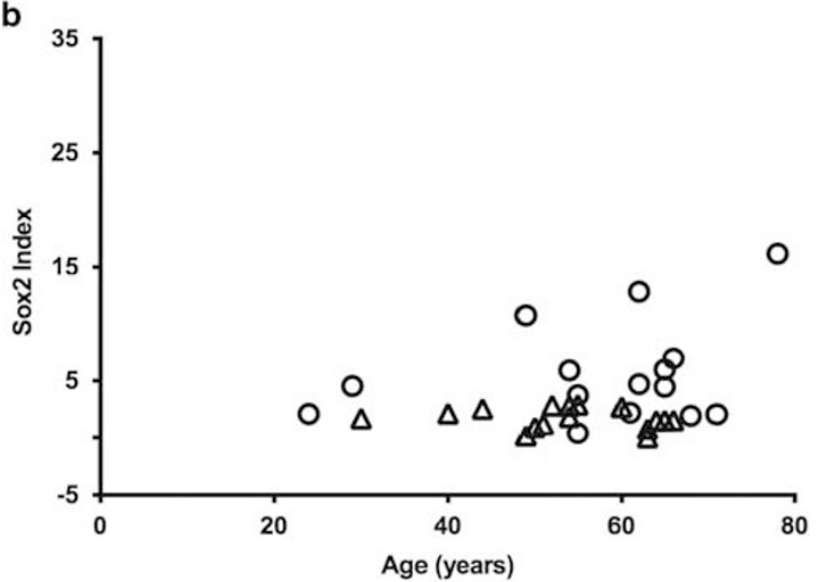

c

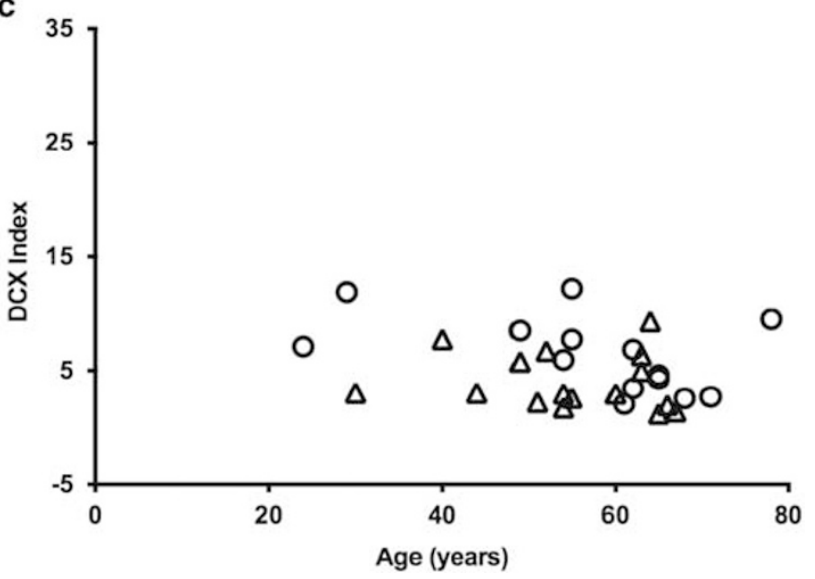

Figure 5 Correlation of markers with age in the SGZ: (a) Ki67-IR significantly decreased with age in controls $(p=0.0470)$ and in all subjects $(p=0.0242)$, but did not reach significance among the alcohol abusers $(p=0.2917)$. (b, c) There were no correlations between Sox2-IR or DCXIR and age of the alcoholic or control subjects. Circles and triangles represent controls and alcoholics, respectively.

et al (2013) counted Ki67 positivities up to several hundreds of $\mu \mathrm{m}$ from the GCL. The reason why investigators have used variable widths when examining SGZ is that this structure is not well defined in the human hippocampus. The CA4 region in primates is much larger than in rodents and the polymorphic layer thins to a narrow SGZ (Amaral et al, 2007), but this zone is still several hundreds of $\mu \mathrm{m}$ wide in humans. When looking for new neurons that may become mature, integrated granule cells, it is reasonable to limit the distance from the GCL in order to avoid counting cells that may have a different fate. In this study we wanted to investigate dividing cells as well as stem/progenitor and immature neurons and therefore decided to set the width to $100 \mu \mathrm{m}$ from the GCL. We expected this width to allow for identification of positive cells for all three markers in such numbers that possible differences between alcoholics and controls could be discovered. This corresponds approximately to the width of 10 granule cells. The topographical distribution of the markers' immunoreactivity in the SGZ showed a general reduction in alcoholics compared with controls in all layers (Figure 4). We also observed that Ki67IR, Sox2-IR, and DCX-IR counts decreased further away outside the $100 \mu \mathrm{m}$ limit.

Although studies on hippocampal neurogenesis have focused on the SGZ, we decided to also count Ki67-IR, Sox2-IR, and DCX-IR cells in the ML of controls and alcoholics, and found immunoreactivity of all the markers in the ML with a significant reduction in alcoholics in Sox2-IR and DCX-IR, but not in Ki67-IR. These results are somewhat difficult to interpret as the possible involvement of cells in the ML in neurogenesis has not been systematically explored. Many different subtypes of ML interneurons and even migrating developing interneurons are recognized (Amaral et al, 2007; Freund and Buzsaki, 1996; Kohler et al, 2011). Studies in mice have revealed that cell division kinetics in the ML is similar to that of the SGZ (Li et al, 2013) and Sox2 is also expressed in the ML of rats (Hattiangady and Shetty, 2008). In humans, Ki67 and DCX immunoreactivity was also shown in the ML (Freund and Buzsaki, 1996; Han et al, 1993; Ribak and Seress, 1983). DCX-IR cells in ML can alternatively represent astrocytes as observed in both adult hippocampus and human neocortex (Verwer et al, 2007), but still be involved in neurogenesis by assisting in the growth of processes that immature neurons in the SGZ will send out into the ML. In addition, in adult mice, molecular layer perforant pathway (MOPP) cells were demonstrated to innervate newly generated granule neurons (Li et al, 2013). This process most likely involves dendrite sprouting that is linked to DCX expression (Rao and Shetty, 2004). Another possibility is that some of the DCX-IR cells in the ML are immature neurons migrating too far into the $\mathrm{ML}$ and not stopping in the GCL, like BrdU-labeled cells may also appear in the ML in experimental animals (Mathews et al, 2010). However, further studies are needed to characterize the cells expressing Sox 2 and DCX in the ML in more detail.

To investigate the effects of alcohol abuse on proliferation, we performed immunohistochemistry against Ki67. The density of Ki67-IR cells in the DG, and specifically in the SGZ, was lower in alcoholics as compared with controls. This is in line with animal studies showing that alcohol inhibits hippocampal cell proliferation (Crews et al, 2006a; He et al, 2005 ) in rodents as heavy binge alcohol does in nonhuman primates (Taffe et al, 2010). Ki67-IR in this study was occasionally colocalized with Sox2-IR that was also significantly reduced in alcoholic DG compared with controls. This finding corroborates the results of studies in nonhuman primates where alcohol treatment reduces the number of cells expressing Ki67 and Sox2 (Taffe et al, 2010). 
In rodents, Sox 2 is expressed in neural stem cells, neuronal progenitor cells, and astrocytes, and this might imply that the results obtained regarding this marker are due to an additive effect of changes in expression of all cell types expressing Sox 2 or because specific cell types are reduced whereas others are unaffected. However, as almost all of the Sox2-IR cells in our study were GFAP negative, we believe that the majority of them rather represent progenitor cells, that is, Type $2 \mathrm{a}$ or Type $2 \mathrm{~b}$ cells in accordance with Knoth et al (2010), who observed that the Sox2-positive cells in the human hippocampus were GFAP negative.

In addition to the reduction among alcoholics of Ki67-IR and Sox2-IR, we found a significant decrease in DCX-IR within the DG and specifically in the SGZ. These results are in line with previous clinically relevant rodent studies of both acute binge and chronic ethanol exposure showing a decrease in DCX-IR (Crews et al, 2006b; He et al, 2005). Most of the DCX cells in the dentate gyrus in our study were also positive for PSA-NCAM (Supplementary Figure S5), further supporting the notion that they possibly represent immature neurons. The coexpression of DCX and PSANCAM is in line with a previous study by Ernst et al (2014), who used the same DCX marker and the same IHC protocol (Ernst et al, 2014). It is important to point out that we performed double staining for various markers only in a few cases and our focus rather was to study the effect of alcohol on Ki67-IR, Sox2-IR, and DCX-IR in isolation and therefore used single immunohistochemistry optimized for best signal to noise for each of them.

Furthermore, in this study we found a negative correlation between Ki67 index values and age of subjects in the two groups, whereas Sox2 and DCX index values showed no correlation with age. Other studies in humans have shown a modest decline in neurogenesis during aging (Knoth et al, 2010; Spalding et al, 2013). This difference could be attributed to the limited number of cases, limited age range, and a considerable level of variability between cases. In rodents an exponential decrease takes place during 36 months of age to reaches a low and constant level thereafter (Ben Abdallah et al, 2010). In humans, a similar decrease in DCX-IR has been shown (Knoth et al, 2010). The majority of the subjects in our study are older adults, making it difficult to observe minor changes with age.

The discrepancy between cases regarding the expression level of markers could depend on chronicity and magnitude of dosing (Vengeliene et al, 2008). We have been able to obtain information regarding drinking frequency, and degrees of drunkenness from next to kin interviews and/or medical charts, but it is very difficult to obtain reliable information about exact amounts consumed. Extrinsic factors, lifestyles such as exercise, health status, and pharmaceutical drugs are additional factors that may affect neurogenesis and explain some of the variation.

None of the subjects included in this study were reported to be active in sports during the past months, although the degree of daily physical activity was difficult to establish.

Epileptic seizures, known to acutely increase neurogenesis, were not reported by relatives or otherwise documented. Pharmaceutical drugs were detected in the blood of several of the subjects. Three of them had low concentrations of selective serotonin reuptake inhibitors (SSRIs): fluoxetine or sertraline. In a study by Boldrini et al (2009), depressed patients treated with SSRIs showed more Ki67-IR cells in the SGZ than nontreated depressed patients, although this difference was much more prominent in the anterior hippocampus than any region further posteriorly. In the same study they also found that the nontreated patients with depression had almost the same densities of Ki67 as nondepressed controls. As our samples are collected from the mid-portion of hippocampus, we believe that the possible impact of this medication is limited. One subject used methylphenidate and pregabalin. Previous studies have shown variable results regarding effects on proliferation and immature neurons in hippocampus when animals have been exposed to these drugs, and there are no human data on this. In three cases opioids were found; in two of these only at low concentrations and in the third case, the concentrations of the drug (tramadol) and its metabolite strongly suggest an acute accidental overdose and not chronic intake, thus making these findings unlikely to have affected the results.

Development of acute to chronic alcohol abuse involves the transitional stages of tolerance and dependence that may be related to changes in cell types involved in the DG neurogenesis. The persisting addictive desire for alcohol that remains following a protracted period of withdrawal has been suggested to be due to long-lasting structural changes in the hippocampus that affect learning processes and particularly memory of the drug euphoria that drives the maladaptive behaviors to increase the risk of relapse (Fibiger and Phillips, 1988; Hyman and Malenka, 2001). The findings in the present study of reduced densities of markers of stem/progenitor cell subpopulations in alcohol abusers are in line with these anatomical-functional associations and hence the affected cells, particularly the putative neural stem cells, may prove to be an important target in future pharmaceutical interventions.

In summary, our results support the notion that on-going alcohol abuse in humans reduces numbers of proliferating cells, stem/progenitor cells, and immature neurons. The decrements in Sox2-IR were more prominent than those of DCX-IR, suggesting that alcohol primarily causes a depletion of the stem/progenitor cells and that immature neurons are probably secondarily affected. Although the markers we have used may not be specific for the cells participating in the generation of new granule cells, our results are compatible with this possibility, and are in agreement with observations of impaired adult hippocampal neurogenesis by alcohol in animal studies and lend further support for the association between hippocampal dysfunction and alcohol abuse.

\section{FUNDING AND DISCLOSURE}

The authors declare no conflict of interest.

\section{ACKNOWLEDGMENTS}

This work was supported in part by NIH/NIDA R01DA031429, Karolinska Institutet KID support, Swedish Research Council, Linnaeus environment grant, and the Swedish National Board of Forensic Medicine. 


\section{REFERENCES}

Altman J, Das GD (1965). Autoradiographic and histological evidence of postnatal hippocampal neurogenesis in rats. J Comp Neurol 124: 319-335.

Amaral DG, Scharfman HE, Lavenex P (2007). The dentate gyrus: fundamental neuroanatomical organization (dentate gyrus for dummies). Prog Brain Res 163: 3-22.

Bayer R, Franke H, Ficker C, Richter M, Lessig R, Buttner A et al (2015). Alterations of neuronal precursor cells in stages of human adult neurogenesis in heroin addicts. Drug Alcohol Depend 156: 139-149.

Belujon P, Grace AA (2011). Hippocampus, amygdala, and stress: interacting systems that affect susceptibility to addiction. Ann NY Acad Sci 1216: 114-121.

Ben Abdallah NM, Slomianka L, Vyssotski AL, Lipp HP (2010). Early age-related changes in adult hippocampal neurogenesis in C57 mice. Neurobiol Aging 31: 151-161.

Boldrini M, Santiago AN, Hen R, Dwork AJ, Rosoklija GB, Tamir H et al (2013). Hippocampal granule neuron number and dentate gyrus volume in antidepressant-treated and untreated major depression. Neuropsychopharmacology 38: 1068-1077.

Boldrini M, Underwood MD, Hen R, Rosoklija GB, Dwork AJ, John Mann J et al (2009). Antidepressants increase neural progenitor cells in the human hippocampus. Neuropsychopharmacology 34: 2376-2389.

Crews F, Nixon K, Kim D, Joseph J, Shukitt-Hale B, Qin L et al (2006a). BHT blocks NF-kappaB activation and ethanol-induced brain damage. Alcohol Clin Exp Res 30: 1938-1949.

Crews FT, Buckley T, Dodd PR, Ende G, Foley N, Harper C et al (2005). Alcoholic neurobiology: changes in dependence and recovery. Alcohol Clin Exp Res 29: 1504-1513.

Crews FT, Mdzinarishvili A, Kim D, He J, Nixon K (2006b). Neurogenesis in adolescent brain is potently inhibited by ethanol. Neuroscience 137: 437-445.

Crews L, Adame A, Patrick C, Delaney A, Pham E, Rockenstein E et al (2010). Increased BMP6 levels in the brains of Alzheimer's disease patients and APP transgenic mice are accompanied by impaired neurogenesis. J Neurosci 30: 12252-12262.

Eriksson PS, Perfilieva E, Bjork-Eriksson T, Alborn AM, Nordborg C, Peterson DA et al (1998). Neurogenesis in the adult human hippocampus. Nat Med 4: 1313-1317.

Ernst A, Alkass K, Bernard S, Salehpour M, Perl S, Tisdale J et al (2014). Neurogenesis in the striatum of the adult human brain. Cell 156: 1072-1083.

Fibiger HC, Phillips AG (1988). Mesocorticolimbic dopamine systems and reward. Ann NY Acad Sci 537: 206-215.

Freund TF, Buzsaki G (1996). Interneurons of the hippocampus. Hippocampus 6: 347-470.

Friocourt G, Koulakoff A, Chafey P, Boucher D, Fauchereau F, Chelly J et al (2003). Doublecortin functions at the extremities of growing neuronal processes. Cereb Cortex 13: 620-626.

Gleeson JG, Lin PT, Flanagan LA, Walsh CA (1999). Doublecortin is a microtubule-associated protein and is expressed widely by migrating neurons. Neuron 23: 257-271.

Han ZS, Buhl EH, Lorinczi Z, Somogyi P (1993). A high degree of spatial selectivity in the axonal and dendritic domains of physiologically identified local-circuit neurons in the dentate gyrus of the rat hippocampus. Eur J Neurosci 5: 395-410.

Hattiangady B, Shetty AK (2008). Aging does not alter the number or phenotype of putative stem/progenitor cells in the neurogenic region of the hippocampus. Neurobiol Aging 29: 129-147.

He J, Nixon K, Shetty AK, Crews FT (2005). Chronic alcohol exposure reduces hippocampal neurogenesis and dendritic growth of newborn neurons. Eur J Neurosci 21: 2711-2720.

He J, Overstreet DH, Crews FT (2009). Abstinence from moderate alcohol self-administration alters progenitor cell proliferation and differentiation in multiple brain regions of male and female $\mathrm{P}$ rats. Alcohol Clin Exp Res 33: 129-138.

Houser CR (2007). Interneurons of the dentate gyrus: an overview of cell types, terminal fields and neurochemical identity. Prog Brain Res 163: 217-232.

Hyman SE, Malenka RC (2001). Addiction and the brain: the neurobiology of compulsion and its persistence. Nat Rev Neurosci 2: 695-703.

Hyman SE, Malenka RC, Nestler EJ (2006). Neural mechanisms of addiction: the role of reward-related learning and memory. Annu Rev Neurosci 29: 565-598.

Kempermann G (2002). Why new neurons? Possible functions for adult hippocampal neurogenesis. J Neurosci 22: 635-638.

Knoth R, Singec I, Ditter M, Pantazis G, Capetian P, Meyer RP et al (2010). Murine features of neurogenesis in the human hippocampus across the lifespan from 0 to 100 years. PLoS ONE 5: e8809.

Kohler SJ, Williams NI, Stanton GB, Cameron JL, Greenough WT (2011). Maturation time of new granule cells in the dentate gyrus of adult macaque monkeys exceeds six months. Proc Natl Acad Sci USA 108: 10326-10331.

Komitova M, Eriksson PS (2004). Sox-2 is expressed by neural progenitors and astroglia in the adult rat brain. Neurosci Lett 369: 24-27.

Kornack DR, Rakic P (1999). Continuation of neurogenesis in the hippocampus of the adult macaque monkey. Proc Natl Acad Sci USA 96: 5768-5773.

Li Y, Stam FJ, Aimone JB, Goulding M, Callaway EM, Gage FH (2013). Molecular layer perforant path-associated cells contribute to feed-forward inhibition in the adult dentate gyrus. Proc Natl Acad Sci USA 110: 9106-9111.

Mathews EA, Morgenstern NA, Piatti VC, Zhao C, Jessberger S, Schinder AF et al (2010). A distinctive layering pattern of mouse dentate granule cells is generated by developmental and adult neurogenesis. J Comp Neurol 518: 4479-4490.

Mullen RJ, Buck CR, Smith AM (1992). NeuN, a neuronal specific nuclear protein in vertebrates. Development 116: 201-211.

Nixon K (2006). Alcohol and adult neurogenesis: roles in neurodegeneration and recovery in chronic alcoholism. Hippocampus 16: 287-295.

Nixon K, Crews FT (2002). Binge ethanol exposure decreases neurogenesis in adult rat hippocampus. J Neurochem 83: 1087-1093.

Rao MS, Shetty AK (2004). Efficacy of doublecortin as a marker to analyse the absolute number and dendritic growth of newly generated neurons in the adult dentate gyrus. Eur J Neurosci 19: 234-246.

Reif A, Fritzen S, Finger M, Strobel A, Lauer M, Schmitt A et al (2006). Neural stem cell proliferation is decreased in schizophrenia, but not in depression. Mol Psychiatry 11: 514-522.

Ribak CE, Seress L (1983). Five types of basket cell in the hippocampal dentate gyrus: a combined Golgi and electron microscopic study. J Neurocytol 12: 577-597.

Scholzen T, Gerdes J (2000). The Ki-67 protein: from the known and the unknown. J Cell Physiol 182: 311-322.

Schwarz TJ, Ebert B, Lie DC (2012). Stem cell maintenance in the adult mammalian hippocampus: a matter of signal integration? Dev Neurobiol 72: 1006-1015.

Spalding KL, Bergmann O, Alkass K, Bernard S, Salehpour M, Huttner HB et al (2013). Dynamics of hippocampal neurogenesis in adult humans. Cell 153: 1219-1227.

Sullivan EV, Rosenbloom MJ, Lim KO, Pfefferbaum A (2000). Longitudinal changes in cognition, gait, and balance in abstinent and relapsed alcoholic men: relationships to changes in brain structure. Neuropsychology 14: 178-188.

Taffe MA, Kotzebue RW, Crean RD, Crawford EF, Edwards S, Mandyam CD (2010). Long-lasting reduction in hippocampal 


\section{Alcohol abuse and hippocampal neurogenesis}

TW Le Maître et al

neurogenesis by alcohol consumption in adolescent nonhuman primates. Proc Natl Acad Sci USA 107: 11104-11109.

Terman A, Brunk UT (2004). Lipofuscin. Int J Biochem Cell Biol 36: 1400-1404.

Vengeliene V, Bilbao A, Molander A, Spanagel R (2008). Neuropharmacology of alcohol addiction. Br J Pharmacol 154: 299-315.

Verwer RW, Sluiter AA, Balesar RA, Baayen JC, Noske DP, Dirven $\mathrm{CM}$ et al (2007). Mature astrocytes in the adult human neocortex express the early neuronal marker doublecortin. Brain 130(Pt 12): 3321-3335.

Vorel SR, Liu X, Hayes RJ, Spector JA, Gardner EL (2001). Relapse to cocaine-seeking after hippocampal theta burst stimulation. Science 292: 1175-1178.

Winner B, Regensburger M, Schreglmann S, Boyer L, Prots I, Rockenstein E et al (2012). Role of alpha-synuclein in adult neurogenesis and neuronal maturation in the dentate gyrus. $J$ Neurosci 32: 16906-16916.

Supplementary Information accompanies the paper on the Neuropsychopharmacology website (http://www.nature.com/npp) 